\title{
The Application of Flipped Classroom in the Teaching of Marine Pharmacology
}

\author{
Chen Yin, Sun Kunlai, Zhao Yuqin, Wang Bin and Qu Youle \\ School of food and pharmacy \\ Zhejiang Ocean University \\ Zhoushan, Zhejiang, China, 316000
}

\begin{abstract}
With the arrival of the information age and the increasing speed of knowledge updating, it has become a challenge faced by the school that how to cultivate students' independent learning abilities to cope with complex problem situations. The teaching model of flipped classroom has attracted the attention of teachers, since it can provide more opportunities for students to actively participate in learning. According to the survey, flipped classroom is at the initial stage of development. More and more teachers attach importance to the satisfaction of students' individual needs and the cultivation of their independent learning abilities, which has become the significant motivation for teachers to improve their teaching methods. At the same time, teaching model of flipped classroom, as an effective way to stimulate students' learning motivation and improve their learning experience, has been recognized by teachers. This paper combines the professional quality and the course characteristics to develop the flipped classroom teaching of Marine Pharmacology on the bas is of micro-video. It designed the course and make students teach after their learning to help them more thoroughly master the knowledge and have more clear knowledge level and more solid knowledge system. "Flipped classroom" has greatly promoted the self-independence of students' learning and improved the flexibility of pharmaceutical chemistry teaching, achieving a good teaching effect. Meanwhile, the required investment of time, energy and technological support in the flipped classroom are the important challenges faced by teachers.
\end{abstract}

Keywords-flipped classroom; Marine Pharmacology; classroom design; Mooc

\section{Introduction OF MARINE PHARMACOLOGY}

Marine Pharmacology is the science of marine drugs, through which active natural marine products could be screened and separated from marine organisms, leading drug compounds could be discovered, and the relationship between drug structure and function could be revealed. What's more, this study could indicate the mechanism of drug action, to develop innovative marine drugs and explore the engineering theory and technology related to marine drugs. Marine Pharmacology is a new cross-applied discipline gradually formed in the process of marine research and development.

As a characteristic course of new subjects, the ultimate goal of Marine Pharmacology is to arouse students' interest and awareness of developing new drugs, to enlighten students for broadening their horizons for marine drug research. It is also meant to improve their ability of integrating theoretical knowledge with practical skills in chemistry, pharmacy, marine biology and so on into the practice of marine pharmacy, also to master the utilization of living marine sources and be familiar with the development of functional products from marine sources. However, there is no systematic and comprehensive introduction of Marine Pharmacology in China, and the categories, objects, and basic ideas of Marine Pharmacology have not been clarified.

As a new subject, marine medicine covers a wide range of subjects, including marine biology, marine chemistry, pharmacy and genetic engineering. Students are required to be familiar with the basic knowledge of marine medicine during a certain period of time, and teachers should focus on the relevant teaching to reflect the connotation and characteristics of marine drug research in our country. In the knowledge system of pharmacy, Marine Pharmacology covers the knowledge of pharmacognosy, natural pharmaceutical chemistry and pharmacology. While studying the characteristics of Marine Pharmacology, students can also apply the knowledge learned into the study of Marine Pharmacology, so that their knowledge could be integrated. In addition, marine pharmacy is a developing science, in particular that the application of biotechnology in marine drug research has greatly promoted the development of marine drug research, consequently, the related research results emerge one after another. The process of learning and mastering the frontier knowledge of marine drug research is also a test and improvement of professional ability.

\section{THE TRANSFORMATION OF FLIPPED CLASSROOM TO CLASSROOM TEACHING}

It can be translated as "Flipped Classroom" or "Inverted Classroom", which means to re-adjust the time inside and outside the class and transfer the decision power of learning from teachers to students. Under such teaching mode, students can pay more attention to the positive learning based on the project during the precious time in the class so as to gain deeper understanding. Its goal is to make students obtain more real learning through practice. The mode of flipped classroom is a part of great education movement, which has the overlapping parts in the meaning with blended learning, inquiry-based learning and other teaching methods and tools. All of them aim at making the learning more flexible and active and helping students to participate more. In the Internet era, students learn abundant online courses through the Internet, 
so they don't have to go to school to be taught by teachers. The Internet, especially the mobile Internet, has spawned the teaching model of "flipped classroom". "Flipped classroom" is a complete subversion of the traditional classroom teaching structure and teaching process based on the printing technology, which will lead to a series of changes of teachers' role, curriculum mode and management mode. The Ministry of Education also encourages to combine with the target and demands of school talents training and constantly innovate the sharing and application mode of in-school and inter-school courses through the application of various methods to the online open courses, such as online learning and the combination of online learning and classroom teaching. Marine Pharmacology, as an interdisciplinary course with distinct characteristics, complex contents and constant development, will bring different and wonderful effects by using the teaching of flipped classroom and rational arrangement and design.

Traditional classroom teaching mode is oriented with teachers in the teaching activities and teaching structure, while students are the passive recipients of knowledge. Knowledge transfer only occurs in the class, and students should digest and absorb the knowledge by themselves, which has hindered students' learning enthusiasm and innovative thinking to some extent. However, the teaching model of flipped classroom based on micro-video can compensate the deficiency of the teaching model of traditional classroom. It puts the transfer of knowledge before the class, and the learning resources are mainly showed in the form of teaching micro-video; and the digestion and absorption of knowledge is put into the class, and students' interest in learning is stimulated through a series of teaching activities in class to improve their innovation abilities. It can be seen that the teaching mode of flipped classroom based on micro-video is necessary and required. Flipped classroom has made great changes with existing teaching model, and it is not simple classroom move, namely, recording the classroom teaching. Especially in the era of mobile Internet, the mobile phone has become the significant tool of connecting the world, so the method of knowledge dissemination has changed a lot. The basic logic of traditional teaching is oriented with teachers. And in the cramming teaching, there are few interactions between teachers and students. So it is hard for teachers to fully understand students and they have no idea whether they are understood or not; and students have few opportunities to learn their real learning conditions so that they are not aware of their position, having the illusion that they have studied well. And in the era of mobile Internet, students' learning is experiencing the great changes with the popularization of high-quality teaching resources. It is difficult for students to focus on their study for a long time due to more abundant information sources; more diversified value orientation and too fragmented learning time and knowledge acquisition. In order to cope with these changes, the transformation of flipped classroom should be oriented with students, which should be presented in all the stages of teaching.

\section{THE DESIGN OF FLIPPED CLASSROOM IN THE DESIGN OF TEACHING APPLICATION OF MARINE PHARMACOLOGY}

In order to highlight the student-centered teaching and learning, it is necessary to realize the blended teaching oriented with flipped classroom. First of all, the most fundamental part is to make online video courses, given the tending fragmented learning time and knowledge acquisition of students. After sorting out the knowledge system of the whole course, the teaching team of Marine Pharmacology science summarized the course content as follows: 1. introduction of course background; 2. marine Chinese medicine; 3. marine natural product; 5 . marine polysaccharide; 6 . pharmacological action of marine drugs; 7. application of marine biotechnology in marine drug development; 8. comprehensive exploitation and utilization of marine medical resources. The 10-15 minutes' video course can be produced through the detailed knowledge points of these eight modules and further integration of knowledge points. Each video tries to achieve the relatively complete and independent knowledge points to meet the demands of fragmented knowledge acquisition. In addition, arrange the knowledge points involved in every video to divide and simplify complex problems to make the questions that can be quickly and objectively assessed, such as choosing the correct item, filling in the blanks or true or false. Students can be directly tested the learning effect after learning the video course to gain the real learning information. On the one hand, students can be urged to improve their attention when learning video courses, instead of doing other things with video open. On the other hand, students can deepen their memory and mastery of knowledge points by working out problems. What's more, the results of the exercise test can be used for teachers to master students' learning conditions to guide classroom teaching.

The teaching model of flipped classroom consists of two parts: pre-class learning and classroom learning. Information technology and activity learning are two vital guarantees for the creation of flipped classroom learning environment in these two processes. The support of information technology and the smooth development of learning activities ensure the creation of personalized collaborative learning environment. Such model can clarify all the parts of the teaching mode of flipped classroom more comprehensively, for instance, students can learn knowledge by watching teaching micro-video. And they can make real-time communication with teachers during their learning. It is possible to establish the part to solve problems and make feedback and assessment in the class. Therefore, the construction of high-quality online courses and flipped classroom cannot be separated from the integration of various teaching forms. At present, flipped classroom teaching still needs the cooperation of classroom teaching. In order to avoid classroom teaching becoming the repetition of flipped classroom, classroom teaching also needs to be well designed. To avoid students losing interest in classroom teaching, especially the network video can selectively publish the video of certain chapters under the combination with classroom teaching, for example, not publishing those rather difficult courses with stronger logic, interdisciplinary content and those knowledge points that can be extended. Taking the natural products of marine microorganisms as an example, a section of 
natural marine products, the characteristics and diversity are related to the biological knowledge of marine microbes. The secondary metabolites of marine microbes are also understandable after the students have studied the chemistry of natural medicine. So the relevant content is based on video courses. However, how to solve the drug source problem by marine microbes is related to the knowledge of special habitat microorganisms, microbial metabolic regulation, co-culture of microbes and microbial engineering and so on. It involves a wide range of knowledge, which is the key content of this chapter, but it needs classroom teaching and emphasis, giving examples and gradually encouraging students to realize the important role of marine microbes in solving the problem of drug source. Furthermore, the biosynthesis and bioreactor of microbial drugs have been a hot topic in the field of marine drug research, which has been developing rapidly in recent years. Understanding students' professional cognition and the grasp of frontier issues will have a good promotion, for this part of the classroom teaching, teachers could encourage students to read literature, guide them to consult the latest foreign literature. After intensive reading, contents of the documents are to be discussed and shared with each other, which not only improves students' ability to consult and translate documents, but also helps them to absorb and solidify the knowledge of documents. For instance, the final chapter of Marine Pharmacology is the comprehensive development and utilization of marine drug resources, the study could have a good review and summary of the whole course. The content of this chapter is mainly taught by students. After the main topics have been assigned, students are grouped and cooperated to consult the relevant information background, to make PPTs and introduce contents in different perspectives, such as Chinese marine medicine, natural marine products, and pharmacological effects and so on. Finally, each group asks questions and reviews within themselves, bringing a good conclusion to the course.

\section{CONCLUSION AND PROSPECT}

Develop the flipped classroom teaching of Marine Pharmacology on the basis of micro-video and design the course. It makes students teach after their learning to help them more thoroughly master the knowledge and have more clear knowledge level and more solid knowledge system. Flipped classroom and blended teaching have brought significant changes to traditional teaching methods in the era of mobile Internet. The original ban on students' taking mobile phones in class may develop into a necessity for mobile phones in class. The development of the new teaching model requires bold exploration and trial, and it needs more investment and design so as to finally play its real role. How to apply this new teaching model to teaching practice is not only in the form but also the problem that most education teaching staff should think and solve. Every teacher should study hard and try to explore a suitable flipped classroom teaching model based on his own teaching style, the actual situation of the school and the nature of the course.

\section{REFERENCES}

[1] Yin Chen, Wang Bin and Qu Youle. Discussion on the Characteristics of education in Marine Pharmacology[J]. Management Observer, 2014(05).

[2] Wang Lingchong, Wu Hao and Liu Rui. Discussion on the course content setting of Marine Pharmacology[J]. Journal of Nanjing University of Traditional Chinese Medicine (the edition of society and science); 2011(03)

[3] Li Le, Sun Yuan and Pan Xinhui. The application of flipped classroom in pharmacy course[J]. Education Science \& Culture Magazine, 2016(20):80-81.

[4] Wang Yanyan, Li Shengli and Huang Lili. The application of flipped classroom in Chinese medicine pharmacology teaching of pharmacy major[J]. Scientific and Technological Innovation,2017(4):77-77.

[5] Chen Ming, Wang Peng and Long Zijiang. Study on the interaction effect on pharmacology teachers and students of flipped classroom[J]. Basic Medicine Education, 2016, 18(7):528-531.

[6] Kou Xiaodi, Yin Fei and Ju Hui. The application of flipped classroom in the bilingual teaching of organic chemistry of pharmacy major[J]. Pharmacologic Education, 2015, 31(6):23-26.. 